[3] Tan TC, Fang H, Magder LS, Petri MA. Differences between male and female systemic lupus erythematosus in a multiethnic population. J Rheumatol. 2012;39:759-69.

[4] Boodhoo KD, Liu S, Zuo X. Impact of sex disparities on the clinical manifestations in patients with systemic lupus erythematosus: A systematic review and meta-analysis. Medicine. 2016;95(29):e4272.

Disclosure of Interest: None declared

DOI: 10.1136/annrheumdis-2017-eular.2323

\section{THU0292 DIAGNOSTIC ACCURACY OF ULTRASOUND AND ULTRASONOGRAPHIC FEATURES OF SALIVARY GLANDS IN PATIENTS WITH PRIMARY SJOGREN'S SYNDROME}

V. Milic, G. Radunovic, N. Damjanov. Institute of Rheumatology, Belgrade, Serbia

Objectives: To analysis ultrasonography (US) changes of salivary glands (SG) in patients with primary Sjogren's syndrome (pSS) and assessment of their accuracy for diagnosis pSS.

Methods: This study included 205 pSS patients (mean age 53.9 \pm 11.5 , disease

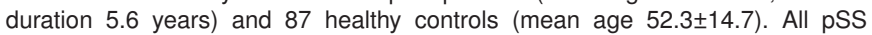
patients fulfilled the AECG diagnostic criteria. The disease activity was measured by EULAR SS disease activity index (ESSDAI). Parotid and submandibular glands on both sides were assessed for size, parenchymal echogenicity and inhomogeneity, posterior glandular border and presence of intraglandular lymph nodes. Inhomogeneity of the salivary glands were graded according to the De Vita scoring system [0) homogenous glands; 1 ) mild inhomogeneous - isolated hypoechogenic areas; 2) evident inhomogeneous - scattered hypoechogenic areas, and/or multiple punctate or linear densities; 3) grossly inhomogeneous - large or confluents hypoechogenic areas, and/or to linear densities, and/or multiple cysts. The global SGUS score (0-6) was the sum of the scores of each pair of salivary glands. Statistical analysis was performed by SPSS v16. Data were compared using $t$-test, $\chi^{2}$ test and Mann-Whitney $U$ test. The optimal cut-off value for SGUS score was calculated as the area under the receiver operating characteristic curve (AUC-ROC).

Results: Xerophtalmia and xerostomia were presented in 185/205 (90.2\%) and $186 / 205(91.2 \%)$, respectively. According to ESSDAI, the majority of pSS patients $88 / 205(43 \%)$ had moderate disease activity. Seventy-eight per cent of pSS patients were anti-SSA antibody positive, 44\% anti-SSB/La antibody positive. Biopsy of LSG was positive in 140/172 (81.4\%) pSS patients. US abnormalities were established in $197(96 \%)$ pSS patients and in $16(18 \%)$ controls $(p<0.0001)$. Pathological sizes of salivary glands were more frequently in pSS patients than controls, $111(54.2 \%)$ vs. $3(3.4 \%)$ patients, respectively $(p<0.0001)$. The echogenicity of the salivary glands was pathological changes in $142(69.3 \%)$ pSS patients and in only $5(5.7 \%)$ control group $(\mathrm{p}<0.0001)$. The pathological glandular border was frequently in pSS patients than control group, $48(23.4 \%)$ vs. $2(2.3 \%), p<0.0001$. No differences were detected between the two groups of patients for enlarged intraglandular lymph nodes. Most of pSS patients had pathological inhomogeneity, 197/205 (96.1\%) vs. 16/85 (18.4\%) in control group $(p<0.0001)$. The median SGUS was significantly higher in pSS patients in comparison with control group [median (range) $4(0-6)$ vs. $0(0-2), p<0.0001]$. Forty-five percent of pSS patients had SGUS score 4 . The SGUS cut-off $\geq 2$ showed specificity of $89.5 \%$ and sensitivity $89.3 \%$. Diagnostic accuracy of the parenchymal inhomogeneity was very good (AUC-ROC 0.89 ), followed by the glandular echogenicity (AUC-ROC 0.81), the glandular size (AUC ROC 0.75), the posterior border (AUC ROC 0.60), and the presence of intraglandular lymph nodules (AUC ROC 0.49), respectively.

Conclusions: Our findings confirm that most of established pSS patients had pathological SGUS features. Among US parameters, parenchymal inhomogeneity was the most discriminant feature for diagnosis of SS. There is the growing evidence that ultrasound should be considered as the useful method for evaluation of salivary glands in pSS patients.

Disclosure of Interest: None declared

DOI: 10.1136/annrheumdis-2017-eular.5046

\section{THU0293 B-CELL RESPONSES TO TYPE I INTERFERON DEFINE DISEASE ACTIVITY IN SLE AND CAN BE MEASURED BY CELL SURFACE TETHERIN (CD317)}

Y.M. El-Sherbiny ${ }^{1,2}$, M.Y. Md Yusof ${ }^{1,2}$, A. Psarras ${ }^{2}$, E. Hensor ${ }^{1,2}$, R. Tooze ${ }^{3}$, G. Doody ${ }^{3}$, M. Wittmann ${ }^{1,2}$, P. Emery ${ }^{1,2}$, E.M. Vital ${ }^{1,2}{ }^{1}$ National Institute of Health Research Leeds Musculoskeletal Biomedical Research Unit, Leeds Teaching Hospitals NHS Trust; ${ }^{2}$ Leeds Institute of Rheumatic and Musculoskeletal Medicine; ${ }^{3}$ Leeds Institute of Cancer and Pathology, University of Leeds, Leeds, United Kingdom

Background: SLE is a Type I interferon (IFN-I) mediated disease with autoreactive $B$ cells. Whole blood interferon-stimulated gene (ISG) expression is used to measure IFN-I status but does not consistently correlate with clinical features and therapy response. ISG expression may be influenced by differing response in individual cell subsets as well as IFN-g. Tetherin is a cell surface protein encoded by the interferon-stimulated gene BST2.

Objectives: To evaluate tetherin expression as a novel cell-specific flow cytometric biomarker for IFN-I response.
Methods: In vitro, we tested response of expression of BST2 and 30 other ISGs to stimulation with IFN-a and IFN-g, as well as dose response of BST2 and tetherin protein in B cells. Sorted cells (Monocyte, T, NK, naïve and memory B, plasmablast) from 8 controls and 10 SLE patients were used to test variation in expression of 31 ISGs between cell subsets and whether tetherin measured by flow cytometry is a cell specific marker for IFN-I response. Samples from 156 SLE patients, 30 ACPA+ANA- RA (DAS28>3.2) patients and 22 healthy controls $(\mathrm{HC})$ were used in 3 clinical validation studies of tetherin vs. ISG score against diagnosis, disease activity (BILAG-2004) and plasmablast repopulation after rituximab. Results: Some ISGs' expression in B cells increased in response to both IFN-a and IFN-g. Others, which included BST2, were selective for IFN-a. An 18-gene ISG score derived from this latter group was calculated for comparison with tetherin.

Results from cell sorting showed that ISG Score was highest in monocytes; other subsets were $75-85 \%$ lower $(p<0.001)$. SLE-associated increase in expression (SLE:HC ratio) varied between 2.56 ( $\mathrm{T}$ cells) to 4.93 (plasmablasts).

Diagnosis: ISG score differentiated HC from SLE with ratio 3.58 (1.94 - 6.61) and large effect size 0.14 (partial eta squared). Using tetherin for cell specific IFN response revealed marked differences between subsets. Monocytes did not differentiate HC and SLE at all with ratio $1.19(0.87-1.61)$ and effect size 0.03 . Memory B cells had medium-large effect size of 0.11 with ratio $1.59(1.21-2.09)$. Comparing SLE and RA the largest effect size was for plasmablast Tetherin at plasmablasts at 0.23 , with ratio $2.20(1.66-2.93)$.

Disease activity: ISG score was associated with cutaneous disease activity (BILAG A/B) but not musculoskeletal (Fig 1). Monocyte tetherin was associated with musculoskeletal disease activity but not cutaneous. Memory B cell tetherin was associated with disease activity in both these organs. Memory B cell tetherin was increased with renal $(p=0.005)$ or haematological $(p=0.005)$ activity with no differences in ISG score for these domains ( $p=0.152, p=0.989$ respectively).

Plasmablast numbers after rituximab were associated with Memory B cell tetherin $(R=0.38, p=0.047)$ but not ISG score $(R=0.24, p=0.219)$.
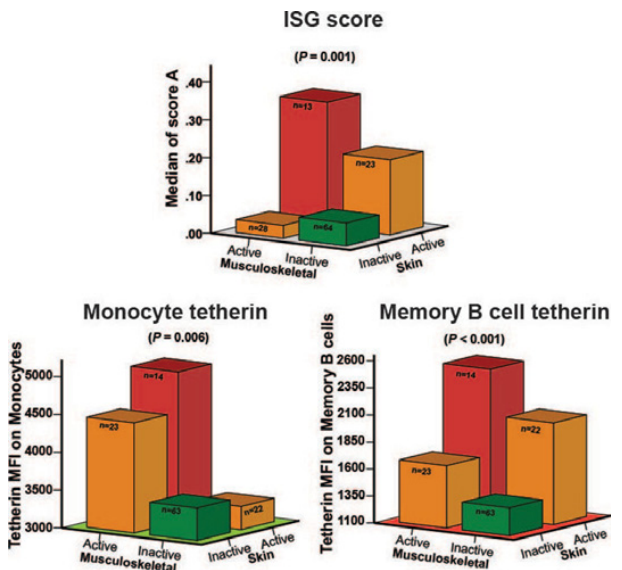

Conclusions: ISG expression in unsorted blood is influenced by IFN-g and cellular composition of the sample as well as IFN-I. Flow cytometric measurement of surface tetherin is a cell-specific assay for IFN-I that avoids these problems. Memory B cell tetherin was better associated with plasmablast numbers and clinical features of disease than monocyte tetherin or ISG score.

Disclosure of Interest: None declared

DOI: 10.1136/annrheumdis-2017-eular.6002

\section{THURSDAY, 15 JUNE 2017 Vasculitis}

\section{THU0294 DIFFERENTIAL PATTERNS OF ATROPHY IN HIPPOCAMPUS AND BRAINSTEM BETWEEN CHRONIC PROGRESSIVE NEURO-BEHCET'S DISEASE AND ALZHEIMER'S DISEASE}

H. Kikuchi ${ }^{1}$, K. Asako ${ }^{1}$, T. Yanagida ${ }^{1}$, H. Kono ${ }^{1}$, S. Hirohata ${ }^{2} .{ }^{1}$ Department of Internal Medicine, Teikyo University School of Medicine, Tokyo; ${ }^{2}$ Department of Rheumatology and Infectious Diseases, Kitasato University School of Medicine, Kanagawa, Japan

Background: Central nervous system involvement is one of the most serious complications in Behcet's disease (BD). This condition is referred to as neuroBehçet's disease (NB) and can be classified into acute type (ANB) and chronic progressive type (CPNB) based upon differences in the clinical course and responses to corticosteroid treatment. Cerebellar ataxia, such as gait disturbances and dysarthria, is one of the representative manifestations in CPNB. Accordingly, brainstem atrophy is frequently observed in CPNB, but not in ANB Notably, progressive neurobehavioral changes mimicking those in Alzheimer's disease (AD) are also frequently observed in CPNB, but these changes cannot be accounted for by brainstem atrophy. 
Objectives: In the present study, volumes of the hippocampus were examined to identify the responsible lesions for neurobehavioral changes in CPNB, and patterns of atrophy in the hippocampus and brainstem were compared between patients with CPNB and AD.

Methods: The subjects were 32 patients, including 13 with CPNB ( 11 males and 2 females, age $51.2 \pm 12.1$ years old [mean \pm SD] $), 13$ with Behçet's disease without NB (non-NB) (10 males and 3 females, age $54.4 \pm 11.4$ years old), and 6 with AD (5 males and 1 female, age $78.8 \pm 7.5$ years old). All patients with BD satisfied the international classification criteria for BD. CPNB was defined as intractable, slowly progressive neurobehavioral changes and/or cerebellar ataxia accompanied by persistent elevation of interleukin-6 of $>20 \mathrm{pg} / \mathrm{mL}$ in cerebrospinal fluid on two different occasions at an interval of at least 2 weeks. All patients with AD satisfied Diagnostic and Statistical Manual of Mental Disorders (DSM)-IV criteria. Brain magnetic resonance imaging (MRI) was obtained from each subject. The areas of the midbrain tegmentum and pons were measured on mid-sagittal sections of T1-weighted images using image analysis software (Image $\mathrm{J}$ ver.1.45: $\mathrm{NIH}$, USA). Severity of gray matter loss in the hippocampal region and whole brain were investigated using Voxel-Based Specific Regional Analysis System for Alzheimer's Disease (VSRAD) software (Eisai Co., Ltd) to determine the degrees of hippocampal region atrophy (Z score) and whole-brain atrophy (WBAI). Thus, the $1 / Z$ score is positively correlated with the hippocampus volume. The ratio of the degree of brainstem atrophy to that of hippocampal atrophy was evaluated by the brainstem area value divided by the $1 / Z$ score (BA/H score) in each patient. Results: The brainstem area was significantly decreased in CPNB $(461.8 \pm 87.3$ [mean $\pm \mathrm{SD}$ ]) compared with that in AD $(661.9 \pm 56.1)$ and non-NB (666.1 \pm 50.6$)$ (Figure $A)$. VSRAD analysis showed that $Z$ score was significantly increased in CPNB $(1.46 \pm 0.70)$ and $A D(3.13 \pm 1.21)$ compared with that in non-NB $(0.77 \pm 0.40)$ (Figure B). Of note, the BA/H score, reflecting the brainstem/hippocampus volume ratio, was much lower in CPNB than in $A D[663.5 \pm 311.8$ vs $2018 \pm 667.6, p=0.001]$ (Figure C).
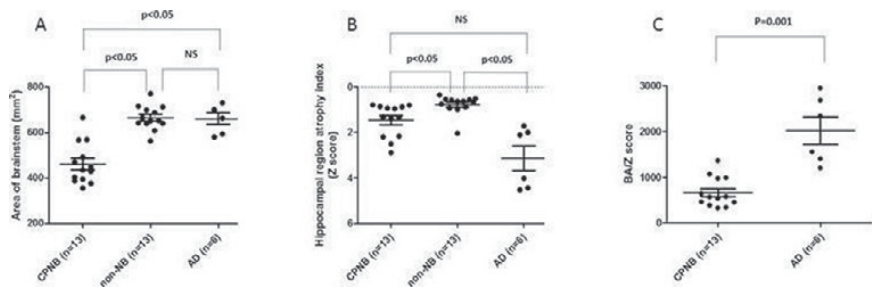

Conclusions: These results indicate that the hippocampus, in addition to the brainstem, is a common target lesion in CPNB, and this accounts for the progressive neurobehavioral dysfunction in this disease. Moreover, the data emphasize that brainstem atrophy is disproportionately greater than hippocampal atrophy in CPNB, in contrast to AD.

Disclosure of Interest: None declared

DOI: 10.1136/annrheumdis-2017-eular.1207

\section{THU0295 CLINICAL FEATURES OF TAKAYASU'S ARTERITIS FROM AN INCEPTION COHORT: EARLY DISEASE IS CHARACTERIZED BY "SYSTEMIC INFLAMMATION"}

F. Alibaz-Oner on behalf of Turkish Takayasu Arteritis Study Group. Rheumatology, Marmara University, School of Medicine, Istanbul, Turkey

Background: There is only retrospective and very limited data for the long term prognosis of Takayasu's Arteritis (TAK), a rare large-vessel vasculitis. In this study, we aimed to present the preliminary results of a Takayasu Inception Cohort settled for long term, prospective follow-up of only newly-diagnosed patients with TAK.

Methods: Patients fulfilling the American College of Rheumatology 1990 criteria for TAK and diagnosed in the last 12 months were included to the study. Patients' data were recorded in an electronic database of an international "Takayasu's Arteritis Registry" requiring baseline and at least annual visits. Data is compared with an historical Turkish cohort previously published (Bıçakçıgil, et al, 2009).

Results: The study included 128 patients (age: $38.9 \pm 13.1$ years, F/M: 112/16) with TAK from 15 tertiary Rheumatology centers in Turkey. The mean symptom duration of patients was 5.2 years at diagnosis. According to the angiographic

Table 1. Clinical characteristics of Inception and Retrospective Cohorts from Turkey

\begin{tabular}{lcc}
\hline & $\begin{array}{c}\text { Inception Cohort } \\
(\mathrm{n}=128)\end{array}$ & $\begin{array}{c}\text { Retrospective Cohort (Bıçakçıgil et al.) } \\
(\mathrm{n}=248)\end{array}$ \\
\hline Constitutional symptoms & $91 / 126(72.2 \%)$ & $163 / 248 /(66 \%)$ \\
Limb claudication & $66 / 106(62.3 \%)$ & $119 / 248(48 \%)$ \\
Carotidynia & $26 / 104(25 \%)$ & - \\
Pulseless & $37 / 104(35.6 \%)$ & $218 / 248(88 \%)$ \\
Musculoskeletal manifestations & $66 / 124(53.2 \%)$ & $104 / 248(42 \%)$ \\
Mucocutaneous manifestations & $32 / 121(26.4)$ & $22 / 248(8.8 \%)$ \\
Respiratory manifestations & $40 / 122(32.8 \%)$ & $22 / 184(12 \%)$ \\
Neurologic manifestations & $58 / 124(46.8 \%)$ & $156 / 248(63 \%)$ \\
Cardiac involvement & $23 / 106(21.7 \%)$ & $141 / 248(57 \%)$ \\
Ophthalmologic involvement & $29 / 125(23.2 \%)$ & $57 / 248(36 \%)$ \\
\hline
\end{tabular}

classification, $59.2 \%$ of the study group had type I and only $17.2 \%$ had type $\mathrm{V}$ disease. When we compared our results to our retrospective cohort (previously published by Turkish Takayasu Arteritis Study Group), constitutional symptoms (72.2\% vs $66 \%$ ) and limb claudication $(62.3 \%$ vs $48 \%$ ) were observed to be more frequent, whereas pulselessness ( $35.6 \%$ vs $88 \%$ ) was less in the inception cohort.(Table 1) Carotidynia was present only in the inception cohort. Similarly, mucocutaneous symptoms also seem to be a feature of newly-diagnosed disease $(26.4 \%$ vs $8.8 \%)$. Regarding comorbidities at diagnosis, the rate of dyslipidemia was $22 \%$, diabetes mellitus $6 \%$, smoking $28.5 \%$ and obesity $(\mathrm{BMI}>30) 15.8 \%$ among TAK patients. All patients were given oral corticosteroid (CS) therapy $(0.5-$ $1 \mathrm{mg} / \mathrm{kg}$ ) at diagnosis, 10 patients $(7.8 \%)$ also having CS pulses. In addition to CSs, 55 patients (43\%) were given methotrexate, 14 patients $(11 \%)$ azathioprine and $5(4 \%)$ cyclophosphomide at disease-onset.

Conclusions: Our results suggest that, in an inception cohort, signs and symptoms of "systemic inflammation" is more prominent in newly-diagnosed TAK patients, whereas vascular extent and damage accumulates during the disease course. The long term follow-up of our inception cohort will better show the actual course and predictors of prognosis in TAK.

Disclosure of Interest: None declared

DOI: 10.1136/annrheumdis-2017-eular.3856

\section{THU0296 THE ROLE OF ULTRASOUND IN THE MANAGEMENT OF GIANT CELL ARTERITIS (GCA) IN ROUTINE CLINICAL PRACTICE}

S. Monti ${ }^{1,2}$, A. Floris ${ }^{3}$, C. Ponte ${ }^{4}$, S. Vaggers ${ }^{2}$, W.A. Schmidt ${ }^{5}$,

A.P. Diamantopoulos ${ }^{6}$, C. Pereira ${ }^{2}$, R. Luqmani ${ }^{2} .{ }^{1}$ Rheumatology, University of Pavia, IRCCS Policlinico San Matteo Foundation, Pavia, Italy, Pavia, Italy;

${ }^{2}$ NDORMS, Rheumatology Department, Nuffield Orthopaedic Centre, University of Oxford, Oxford, United Kingdom; ${ }^{3}$ Rheumatology, University Clinic and AOU of Cagliari, Cagliari, Italy; ${ }^{4}$ Rheumatology, Hospital de Santa Maria, Rheumatology research Unit Instituto de Medicina Molecular, Lisbon Academic Medical Centre, Lisbon, Portugal; ${ }^{5}$ Rheumatology, Immanuel Krankenhaus Berlin, Medical Centre for Rheumatology Berlin-Bu, Berlin, Germany; ${ }^{6}$ Rheumatology, Hospital of Southern Norway Trust, Kristiansand, Norway

Background: Colour Doppler sonography (CDS) is an emerging diagnostic tool for giant cell arteritis (GCA), however, its use in routine practice is still not widespread.

Objectives: To develop a protocol using CDS and explore its value in the routine care of patients with GCA.

Methods: We developed a structured scanning protocol for CDS of temporal and axillary arteries (total of 8 anatomical sites scanned per patient) based on previously published methods. We tested the protocol on consecutive patients referred to a single rheumatology centre, with suspected or established GCA, between July 2014 and September 2016. We defined a positive scan by the presence of halo in at least one branch of a temporal artery (TA) or one axillary artery (AX). We report data from the first 293 consecutively scanned cases.

Results: We assessed 293 patients (mean age $72 \pm 10$, female/male 196/97), of whom 118 had clinically confirmed GCA. Amongst new referrals with confirmed diagnosis of GCA, $44 \%$ had a positive scan; two patients with a positive scan did not have GCA. $82 \%$ of new referrals patients showed exclusive TA involvement, $25 \%$ concomitant AX, and $4 \%$ exclusive AX involvement. High-dose glucocorticoid therapy had already been started in $78 \%$ of these patients for an average of $17 \pm 33$ days. Amongst this group, the sensitivity of CDS was $46 \%(95 \%$ IC $37 \%>55 \%)$, specificity $98 \%(95 \% \mathrm{Cl} 93 \%>99.8 \%)$, positive predictive value $96 \%$ $(88 \%>99.6 \%)$, and negative predictive value $60 \%$ (95\% IC $52 \%>68 \%$ ). During the period of observation, the rate of temporal artery biopsies (TAB) decreased significantly from $42 \%$ to $25 \%(p=0.002)$. During follow up, CDS was positive in $21 \%$ of 89 routine scans in asymptomatic individuals, compared to $58 \%$ in patients with confirmed clinical flares $(45 \%$ of whom had negative inflammatory markers). Over time, the number of halos per patient reduced; only new or flaring patients showed a halo in $>4$ sites. Halo size at the TA did not change significantly (average thickness $0.6 \pm 0.1 \mathrm{~mm}$ ), however, the size of AX artery halos significantly reduced from first referral $(1.6 \pm 0.4 \mathrm{~mm})$ to follow up $(1.4 \pm 0.2, p=0.01)$ or during subsequent flares $(1.4 \pm 0.2, p=0.02)$.

Conclusions: We have developed and tested a standardised methodology for CDS evaluation of GCA. CDS provides a high positive predictive value for a diagnosis of GCA in unselected patients from routine clinical practice, although prior high dose glucocorticoid therapy is likely to reduce its sensitivity. CDS allows for a significant reduction of TAB. We explored the role of CDS to detect disease flares and demonstrated a significant reduction in the extent of abnormalities, and of the size of halo of the AX arteries during follow up or flares. These findings could have a significant impact on the management of patients with suspected and confirmed GCA.

Disclosure of Interest: None declared

DOI: 10.1136/annrheumdis-2017-eular.4063 